\title{
Underlying factors in the willingness to receive and barriers to receiving the COVID-19 vaccine among residents in the UK and Nigeria: a qualitative study
}

\author{
Ifeanyichukwu Anthony Ogueji ${ }^{1}$ (D) Maia Makeda Okoloba ${ }^{2,3}$
}

Accepted: 11 November 2021

(c) The Author(s), under exclusive licence to Springer Science+Business Media, LLC, part of Springer Nature 2021

\begin{abstract}
Qualitative data on the factors underlying the willingness to receive and barriers to receiving the COVID-19 vaccine were scant in the literature. Therefore, the authors employed a qualitative design with a heterogeneous sample of 60 residents (age range $=18-79$ years) in the UK and Nigeria to explore the factors underlying their willingness to receive and barriers to receiving the COVID-19 vaccine. The thematic analysis was employed to analyze data. The results revealed that only a small number of the participants had received the COVID-19 vaccine; they experienced soreness and itchiness, and their motive for receiving the vaccine was its availability. The participants who had not received the vaccine reported the following as determinants of their willingness to receive the vaccine: "concerns about the side/adverse effect", "the perceived benefit of receiving the vaccine", "mistrust (in the pharmaceutical companies that produced the vaccine, the vaccine itself, or governments)", "the need for clarity of information on the vaccine", and "moral obligation to receive the vaccine". The participants who had not received the vaccine further reported the following as other barriers that limit them from receiving the vaccine: "unavailability of the vaccine in the country of residence", "non-membership to a high-risk group", and "membership to a minority group". In terms of what governments can do to encourage public uptake of the vaccine, many participants reported: "provide clear information on the COVID-19 vaccine", "endorsement by public figures", "make the vaccine free to receive", "introduce rewards and punishments", and "honesty from governments". Implications for practice are highlighted.
\end{abstract}

Keywords COVID-19 vaccine $\cdot$ Willingness to vaccinate $\cdot$ Barriers to vaccinate $\cdot$ General public $\cdot$ The UK $\cdot$ Nigeria

\section{Introduction}

The coronavirus disease 2019 (COVID-19) continues to threaten the world since its first report in Wuhan, China in December 2019. As the COVID-19 is now a pandemic and continues to threaten global health (Chen et al., 2020; Coulthard, 2020; Cuschieri, 2020; Dodd et al., 2020; Ogueji et al., 2021a, 2021b; Okoloba et al., 2020; Qian \& Jiang, 2020; Robinson \& Daly, 2020; Robles-Bello et al., 2020; Shaaban et al., 2020; Williams et al., 2020; World Health

Ifeanyichukwu Anthony Ogueji

ifeanyiogueji21@gmail.com

1 Department of Psychology, Faculty of the Social Sciences, University of Ibadan, Ibadan, Oyo State, Nigeria

2 School of Psychology, University of Sussex, Brighton, UK

3 Surrey and Borders Partnership NHS Foundation Trust, Redhill, UK
Organization, 2020), clinical trials have been ongoing to develop vaccines against the disease (Bokemper et al., 2020; Danchin et al., 2020; Giubilini, 2020; Haynes et al., 2020; Kaur \& Gupta, 2020; Lazarus et al., 2020; McClung et al., 2020; Pogue et al., 2020; Su et al., 2020; Verger \& Dubé, 2020; Zhu et al., 2020). According to the media report, many countries have implemented a vaccine distribution plan that prioritized at-risk groups, such as the elderly population (Beaumont, 2020). And as of 05 February 2021, when this paper was written, over 12.3 million doses of the COVID-19 vaccine have been administered globally with most developed countries reporting higher uptake than less developed countries (Ritchie et al., 2021).

In the growing body of knowledge on the COVID-19 vaccine, there is evidence which reports that the uptake of the COVID-19 vaccine is determined by one's willingness and many barriers (of which the underlying factors in the willingness and barriers are unknown from the perspectives of the general public). For instance, in a sample of 1971 
American adults, a cross-sectional survey revealed that the willingness to uptake the COVID-19 vaccine was significantly associated with factors such as the vaccine efficacy strength, protection duration, the vaccine country of origin, and endorsement by the Food and Drug Administration (FDA) (Kreps et al. 2020). These design and findings are indicative that the American study was limited from exploring the underlying factors in the willingness to receive the COVID-19 vaccine from the general public's perspectives given its quantitative methodology.

In a different study, a cross-sectional survey with Malaysian residents reported that most participants were willing to receive the COVID-19 vaccine; however, affordability, educational levels, and occupation were potential barriers limiting them from receiving the vaccine (Wong et al., 2020). An international cross-sectional survey with 1541 caregivers also revealed that most participants agreed to vaccinate their children once the COVID-19 vaccine was out (Goldman et al., 2020). In the United Kingdom (UK), an online survey with 5114 adults found that the COVID-19 vaccine hesitancy was relatively evenly spread across the population (Freeman et al., 2020). Nigerian scholars added by reporting from a sample of 465 respondents, that $59.8 \%$ supported the COVID-19 vaccine (Enitan et al., 2020). Although these studies (Enitan et al., 2020; Freeman et al., 2020; Wong et al., 2020) provide empirical data to support the uptake of the COVID-19 vaccine, these studies were quantitative in design. Therefore, the researchers were limited from conducting an in-depth probe into the factors that underly the willingness to receive and barriers to receiving the COVID-19 vaccine. Thus, the value in carrying out a qualitative study in this light.

It is worth mentioning that the UK was among the hardest-hit by the COVID-19 pandemic (World Health Organization, 2020), and Nigeria was also hit by the pandemic; therefore, residents in these countries deserve attention to the underlying factors in their willingness to receive and barriers to receiving the COVID-19 vaccine. Understanding the underlying factors is expected to inform practical interventions for governments and stakeholders, and potentially mitigate the adverse health impact that is posed by the threat of subsequent waves of the pandemic (Beaumont, 2020; Ritchie et al., 2021). Further, the need to conduct studies that inform public health messages aimed at strengthening COVID-19 vaccination in different nations including European and African nations has been highlighted by scholars (e.g., Murphy et al., 2021; Neumann-Böhme et al., 2020; Nkengasong et al., 2020).

On this premise, our current study aimed to qualitatively explore the factors that underly the willingness to receive and barriers to receiving the COVID-19 vaccine from the perspectives of residents in the UK and Nigeria. Our current study aimed to complement existing quantitative data reported in the growing body of knowledge on the COVID19 vaccine since quantitative and qualitative data have been recommended to go hand-in-hand to inform educated decisions about a problem (Wisdom \& Creswell, 2013). The findings from our current study will contribute to the development of targeted intervention programs that strengthen the uptake of the COVID-19 vaccine, thus protecting public health in the long term.

Based on existing literature on the uptake of the COVID19 vaccine, we believe that our study is the first to qualitatively explore the underlying factors in the willingness to receive and barriers to receiving the COVID-19 vaccine, and through this, report intervention programs that governments can implement to increase uptake from the perspective of the general public. Theoretically, we employed the health belief model (HBM) to argue that our participants' willingness to receive the COVID-19 vaccine may be determined by factors such as the perceived benefit associated with the vaccine or other similar factors and that barriers to receiving the COVID-19 vaccine can determine their actual uptake of the vaccine (Green et al., 2020).

To achieve the aim of our current study, we asked: (1) In the words of residents in the UK and Nigeria who received the COVID-19 vaccine, what was their experience of receiving the vaccine, and what motivated them to receive the vaccine? (2) From the perspective of residents in the UK and Nigeria who have not received the COVID-19 vaccine, what factors can determine their willingness to receive the vaccine? (3) From the perspective of residents in the UK and Nigeria who have not received the COVID-19 vaccine, apart from the factors that determine their willingness, what barriers limit/hinder them from receiving the vaccine? Lastly, (4) From the perspective of residents in the UK and Nigeria (both those who received and have not received the COVID19 vaccine), what can the governments do to increase the uptake of the COVID-19 vaccine?

\section{Method}

\section{Design}

We employed a qualitative open-ended design to achieve the aim of our study. In this design, participants responded to open-ended questions (i.e., they were free to provide responses without restriction) which enabled us to understand the underlying factors in the willingness to receive and barriers to receiving the COVID-19 vaccine in the words of the participants (Elliott \& Timulak, 2005). This design was most appropriate given the aim of our study and the research questions that our study aimed to answer. Additionally, as mentioned in the "introduction" section of this paper, that a qualitative report was lacking in the literature of the uptake 
of the COVID-19 vaccine, the current study employing a qualitative open-ended design was thus imperative enough.

\section{Participants}

To be included in the current study, we required that participants were adult (18 years or above) residents in the UK or Nigeria, could communicate (written \& verbal) using the English language, and could access our online survey from anywhere in the UK or Nigeria. A snowball technique was employed across social media (e.g., Facebook, WhatsApp, Twitter, LinkedIn, etc.) to recruit participants from various groups for the UK or Nigerian residents. We employed the support of our research team members, friends, colleagues, and associations (e.g., British Psychological Society - Health Psychology Division) to distribute our survey link, and a statement was included in the survey that requested participants to roll out the survey link to their counterparts. Participants were recruited from 05 February 2021 to 12 February 2021 when data saturation occurred (Elliott \& Timulak, 2005). Only one response per participant was permitted.

Sixty (30 UK residents and 30 Nigerian residents) responses were received in our online survey, and all responses were complete. The UK residents comprised 13 males, 15 females, and two participants who preferred not to say their gender, while Nigerian residents comprised 12 males and 18 females. In total, participants' ages ranged from 18 to 79 years. In terms of the occupation of participants, many (37 participants) reported being employed with jobs such as engineer, university lecturer, web developer, customer support, cardiac physiologist, mental health support worker, community health researcher, inventory manager, tailor, firefighter, or psychologist. Further, 20 participants reported their occupation as students, two participants reported as retirees, and one participant reported as unemployed.

Participants reported diverse ethnic identities (e.g., Asian/ Asian British, Black/African, Black British, Caribbean, Caucasian, Hausa, Igbo, White British, White Irish, Yoruba, or other). In terms of highest education attained, 35 participants reported to have attained higher education (e.g., Bachelor's degree, Master's degree, or Ph.D.), while 25 reported having attained below higher education (e.g., high-school).

\section{Materials}

Data were collected using Google Forms. We designed an online data collection form that elicited demographic information (gender, age, country of residence, highest education attained, occupation, and ethnicity). This was followed by six open-ended questions that sought to achieve our research aims and objectives. These questions were generated through consultation with the theory and the literature (Enitan et al., 2020; Freeman et al., 2020; Green et al., 2020; Reiter et al., 2020; Warren et al., 2020; Williams et al., 2020; Wong et al., 2020), and two health practitioners (one health psychologist and one public health expert). Above the open-ended questions, we highlighted that participants should be elaborative with their responses given the qualitative design of our study. This was imperative to highlight to reduce the shortcoming that could be associated with collecting qualitative data using online data collection forms. The open-ended questions were:

1. Have you received the COVID-19 vaccine? If yes, please tell us about your experience.

2. If you have received the COVID-19 vaccine, please tell us what motivated you to receive it.

3. If you have not received the COVID-19 vaccine, in your own words, discuss what factors can determine your willingness to receive the vaccine. Please, discuss it extensively.

4. If you have not received the COVID-19 vaccine, apart from the factors that determine your willingness to receive the vaccine, describe what barriers potentially limit you from receiving the vaccine. Elaborative description, please.

5. How do you think governments can encourage the general public to receive the COVID-19 vaccine? Please, provide an elaborate response.

6. Is there anything else on your willingness to receive and barriers to receiving the COVID-19 vaccine that you would like to discuss? If yes, please discuss it extensively.

All questions were compulsory (therefore, participants responded "not applicable" where appropriate. For instance, participants who had not received the vaccine responded "not applicable" to the first question, etc.). Participants who responded "yes" to question 6 had their responses analyzed and merged to any similar theme that was previously identified. Before conducting this study, the questions were piloted with five laypersons recruited from the WhatsApp contact of the authors, and these persons were excluded from the main study. The piloting revealed "blind spots" in our questions that required amendments, and after amending the questions, our main study was conducted.

\section{Data Collection Procedure and Process of Data Analysis}

The co-author (Ogueji) wrote a proposal that was submitted by both authors for institutional ethical review. Following approval, we proceeded to data collection by individually 
posting the survey link to social media (e.g., Facebook, Twitter, WhatsApp, LinkedIn, etc.) to enable access to participants with diverse socio-demographic backgrounds. We collected data from our respective locations in the UK and Nigeria.

The Google Forms explained the research aims and objectives and included an online consent form. Participation in this study was voluntary, and the Google Forms was an anonymous one. Further, a statement assuring participants of confidentiality was included in the survey. We also included a statement that informed participants of their rights to withdraw their participation at any time without implication(s). On the last page, the contacts of psychological helplines that offered free psychosocial support services to clients from anywhere in the world were included, and we stated that participants were free to contact the helplines if they needed any psychosocial support in the face of the pandemic. While data collection was ongoing, we met virtually on two days, each at 9.00 am UK time to openly discuss the demographic diversity and responses of participants to identify potential themes. It was when we observed data saturation that we ended data collection. Following data collection, we exported data for cleaning and embarked on a thematic analysis (TA) using a data-driven method.

Both authors of this paper are members of an international research team. Therefore, we had experiences carrying out qualitative studies (e.g., Ogueji et al., 2021b; Okoloba et al., 2020; Okoloba \& Ogueji, 2020). Further, we both needed to carry out the thematic analysis given the fact that we had first-hand knowledge of participants' responses through the meetings that we attended while data collection was ongoing (Sidhu et al., 2020). Further, both authors have attended and organized several qualitative research workshops in Nigeria and the UK. Additionally, the co-author (Ogueji) has broad experience co-supervising qualitative researches. Before conducting the thematic analysis, we employed bracketing by openly reading the literature and discussing areas of our knowledge and experiences that could potentially influence the thematic analysis (Tufford \& Newman, 2012). Any identified knowledge or experience was written down and openly discussed on how to control for it.

In the thematic analysis, we engaged in immersion with the data by reading and re-reading the responses of participants to identify recurring themes. After themes were created, we extracted illustrative quotation(s) that endorsed each theme and placed it under the theme appropriate for it. We read and re-read each theme and quotation under it to ensure that each theme and quotation under it were appropriate for each other. After this, we openly discussed our results and defined and refined the theme where necessary. As a part of the quality check, after data analysis, the participants were asked to give feedback (validation of analysis by participants). Participants were presented with our findings and asked to express their general views about our findings and confirm if our findings represent their views.

Five participants (two UK and three Nigerian residents) were asked to give feedback based on the recommendations for qualitative studies by Elliott and Timulak (2005), and the five participants provided positive feedback, for instance, a participant's feedback was this - "I absolutely love the fact that the study focused on more than one nation, and it's so amazing how both nations represented have almost similar themes." Further, two external qualitative researchers were invited to validate our analysis (validation of analysis by experts), and any disagreement was resolved by reworking the themes. Our study followed the consolidated criteria for reporting qualitative research in healthcare (COREQ) (Booth et al., 2014).

\section{Results}

The thematic analysis was conducted according to the questions asked; therefore, the results were presented in the order of questions asked. Every response endorsing the themes was labeled with the endorsing participant's gender, age, and country of residence.

Question 1: Have you received the COVID-19 vaccine? If yes, please tell us about your experience.

\section{Adverse Bodily Reaction}

Two participants reported to have received the COVID-19 vaccine, and from their responses, the theme of the adverse bodily reaction was found. A participant residing in the UK had this to report:

“...Yes, I received the first dose, and you know what? My hand was sore the day after. I am scared because I do not want to get sick from this sore..." (Female, 40 years, the UK).

Another UK resident supported this theme by describing it as follows:

"I've gotten my first dose...On the first day of the vaccination, my whole arm hurt; the second day, it was itching." (Female, 63 years, the UK).

Question 2: If you have received the COVID-19 vaccine, please tell us what motivated you to receive it.

\section{Availability of the Vaccine}

From the same two participants above, the availability of the COVID-19 vaccine was found as the core drive for receiving 
the COVID-19 vaccine. Although the vaccine was available by chance, for instance, a participant reported:

"Currently, only key populations are called to be vaccinated. However, sometimes there are 'leftovers' that need to be used immediately or disposed of. I managed to get vaccinated by hearing about a nursing home with several vaccinations to be disposed of by the end of the day." (Female, 40 years, the UK).

The second participant added to this theme as follows:

"I have a lot of health conditions, so I was expecting to wait and see what happened with other people who were vaccinated, but then my school said they had doses...so I decided to be one of the first 'lab rats', instead of one of the last." (Female, 63 years, the UK).

Question 3: If you have not received the COVID-19 vaccine, in your own words, discuss what factors can determine your willingness to receive the vaccine. Please, discuss it extensively.

\section{Concerns about the Side/Adverse Effect}

Out of 58 participants who had not received the vaccine, most (about 40) participants were concerned about the side/ adverse effects of the vaccine, and this was reported as a factor that determined their willingness. A resident in Nigeria endorsed this theme by mentioning that there are currently no proofs on the side effect of the vaccine, as well as its efficacy.

"Yet to be provided proofs of its efficacy and zero or no side effects" (Male, 35 years, Nigeria).

A UK resident supported this theme as follows:

"I am reluctant to get the vaccine because there is uncertainty about whether the vaccine will cause fertility issues. I would like to have children in the future and I wouldn't like the vaccine to inhibit my ability to do so. Also, they have not studied the long-term effects of the vaccine. There could be other effects that don't manifest until 10 years and we don't know about it." (Female, 23 years, the UK).

\section{The Perceived Benefit of Receiving the Vaccine}

Many participants (about 31) reported the benefit associated with receiving the vaccine as another factor that determined their willingness to receive the COVID-19 vaccine. Theoretically, we argued that this theme aligned with the HBM which we employed to argue in the "introduction" section of this paper that the perceived benefits of receiving the vaccine, as well as other similar factors can contribute to determining the general public's willingness to receive the vaccine.

A resident in the UK supported this theme as follows:

“...The idea that if I get the vaccine, I'll be more protected and thus my family will be more protected, is a factor that makes me more willing to receive the vaccine. The idea that if as many people receive the vaccine as quickly as possible, it will help society get back to normal, is a factor that makes me more willing to receive the vaccine." (Female, 23 years, the UK).

A resident in Nigeria added by mentioning the high risk of exposure at his workplace.

"My willingness is because of the high risk of exposure at work (i.e., the vaccine will protect me at work)."

(Male, 40 years, Nigeria).

\section{Mistrust (in the Pharmaceutical Companies that Produced the Vaccine, the Vaccine itself, or Governments)}

Many participants who had not received the vaccine highlighted mistrust in the pharmaceutical company that produced the vaccine, the vaccine itself, or the government as the core factor that determined their willingness to receive the vaccine.

A resident in the UK expressed mistrust in the pharmaceutical company producing the vaccine as follows:

“...When Pfizer came out, their company's reputation wasn't great so I don't know why we are trusting companies with a bad reputation to create vaccinations..." (Preferred not to say gender, 23 years, the UK).

A resident in Nigeria also described:

"To be honest, I am quite doubtful of the vaccine and the Nigerian government. I think a lot of the general population is too. However, the COVID-19 vaccine may be one that everyone will have to succumb to, considering the threat of subsequent COVID-19 waves." (Female, 71 years, Nigeria).

Another resident in the UK endorsed this theme by reporting her mistrust of health service providers and the government. Uniquely, she narrowed her mistrust to the management of Black patients. From a practical side, this may suggest the need for culturally specific consideration in the development of policies and programs that are aimed at encouraging the public uptake of the COVID-19 vaccine.

“...Lack of trust of health services and the governments when managing Black patients...." (Female, 27 years, the UK). 


\section{The Need for Clarity of Information on the Vaccine}

Some participants described the need for clarity of information on the COVID-19 vaccine as another factor that determined their willingness to receive the vaccine. For instance:

“...Accurate information needs to be provided on the vaccine, who is making these vaccines, and how reliable the pharmaceutical companies are (Why have they not mentioned a lot about this? And does this vaccine mean we definitely won't get COVID-19 even if it is a new variant?)" (Preferred not to say gender, 23 years, the UK).

Participants in Nigeria also reported as follows:

"Currently, I am not yet decided about this vaccine. I would like to be better informed about these vaccines." (Female, 47 years, Nigeria).

"There needs to be a sustained effort to combat the misinformation being circulated on social media regarding vaccines..." (Male, 55 years, Nigeria)

\section{Moral Obligation to Receive the Vaccine}

Some participants highlighted that they feel morally obliged to receive the COVID-19 vaccine; therefore, this was a factor that determined their willingness. Illustrative responses endorsing this theme are below:

"I am willing to receive the vaccine because I think it is a responsible thing to do for self, family, friends, and society as a whole..." (Female, 79 years, Nigeria) "I am willing to receive the vaccine because I feel morally obliged but if I felt I could choose without feeling guilty, I wouldn't receive it..." (Female, 22 years, the UK).

Question 4: If you have not received the COVID-19 vaccine, apart from the factors that determine your willingness to receive the vaccine, describe what barriers potentially limit you from receiving the vaccine. Elaborative description, please.

\section{Unavailability of the Vaccine in the Country of Residence}

Most participants reported that the COVID-19 vaccine was unavailable in their countries. This, therefore, was a potential barrier to receiving the vaccine among them. A male resident in Nigeria reported this:

"The vaccine is not available here, and we don't know when it will be available ..." (Male, 36 years, Nigeria).
Residents in the UK also described as follows:

"It is not fully available for everyone here..." (Male, 57 years, the UK)

"The vaccine is not so available and it feels contested here" (Female, 30 years, UK).

\section{Non-membership to a High-Risk Group}

Many participants reported that they do not belong to high-risk groups, and this could limit them from receiving the vaccine even when it is available. Illustrative responses supporting this theme are below:

"When I've looked at when I'm likely to get the vaccination given my demographic and risk profile, I probably won't get this until near the end of 2021 or the beginning of 2022. This slow rollout of the vaccination could be for many reasons e.g., staff availability; the number of vaccines procured; problems with storage/transportation, etc." (Female, 49 years, the UK).

The non-membership to a high-risk group was reinforced by participants:

"I am not within the requirement yet (not classified as high-risk), so I am waiting to be given the goahead." (Female, 18 years, the UK).

"Although the vaccine is unavailable in Nigeria, I am not classified as high-risk, so this could be a barrier..." (Female, 52 years, Nigeria).

\section{Membership to a Minority Group}

Some participants submitted that membership to minority groups was another barrier that could potentially limit them or others from receiving the vaccine when it becomes available. For instance:

"Developing nations will most likely receive the vaccines after other more developed nations. Even when it becomes available in Nigeria, the process of distribution is likely to favor a certain class of people over others (e.g., based on social class)" (Male, 36 years, Nigeria).

Another participant also supported this theme by highlighting as follows:

“...Sterilization histories and discrimination (e.g., AIDS crisis) and the presence of racism within the healthcare system can affect the rollout of the vaccine" (Female, 45 years, the UK) 
Question 5: How do you think governments can encourage the general public to receive the COVID-19 vaccine? Please, provide an elaborate response.

\section{Provide Clear Information on the COVID-19 Vaccine}

Most participants stated that the governments should provide clear and accurate information to the general public on the COVID-19 vaccine to encourage its public uptake. For instance, a resident in Nigeria suggested that governments should provide more information through countryspecific research.

"Governments can conduct independent countryspecific research to provide more information and convince the people" (Male, 35 years, Nigeria)

Participants in the UK supported the need for governments to provide clear information on the COVID-19 vaccine by highlighting the importance of vaccine question-andanswer sessions (Q\&A) and communication in layman's term:

"Governments can do this by getting virologists to do a vaccine question and answer session $(Q \& A)$ that answers all questions the general public may have. By being completely honest about all the information they know about the vaccine (both the good \& bad) so people can make an informed decision. By confronting all the vaccine myths e.g., about how the vaccine can change your DNA." (Female, 23 years, the UK).

"To provide practical information in layman's terms. On Facebook, I am seeing a lot of people every day stating they will not be taking the vaccine, none of these people have any qualifications or experience in healthcare, they are just simply scared, and angry at their government's response to the pandemic. Governments need to explain in colloquial language what the vaccine is and how it will help. The National Health Service (NHS) Scotland issued a pamphlet to every household explaining the vaccine, which was very helpful." (Female, 25 years, the UK).

\section{Endorsement by Public Figures}

Many participants recommended that the governments can encourage the uptake of the COVID-19 vaccine by publicizing public figures endorsing the vaccine. A resident in the UK supported this theme as follows:

"They (governments) should publicize themselves, and their children receiving the vaccine and let us know how they got on first and prove we are all getting the same vaccine" (Male, 55 years, the $\mathrm{UK}$ ).
A Nigerian resident added as follows:

"By the officials taking it publicly, this can encourage people to receive it." (Male, 40 years, Nigeria).

\section{Make the Vaccine Free to Receive}

Many participants highlighted that governments can also increase the number of people receiving the COVID-19 vaccine by making the vaccine free to the general public. For instance, a resident in Nigeria submitted this:

"The governments should give it to people free of charge..." (Female, 46 years, Nigeria).

From the UK, a participant highlighted:

"The governments should keep it free, because when people are required to pay for it, they may be discouraged" (Female, 29 years, the UK).

\section{Introduce Rewards and Punishments}

Some participants recommended that the governments should attach certain rewards or punishments to the COVID19 vaccine. From the perspectives of these participants, this would enhance the public uptake of the vaccine.

A resident in the UK supporting this theme had this to report:

"Governments should link vaccination to greater freedoms/fewer restrictions" (Male, 51 years, the UK).

A resident in Nigeria also described as follows:

"Governments should attach benefits for those who were vaccinated, and punishments to those refusing vaccinations...." (Male, 41 years, Nigeria).

\section{Honesty from Governments}

Some participants recommended that governments should employ honesty to encourage the general public to receive the vaccine. Illustrative responses endorsing this theme were presented below:

"The governments should be very truthful/honest with their whole conscience" (Male, 38 years, the UK)

"Let them (the governments and pharmaceutical companies producing the vaccine) be very honest and more transparent about it..." (Female, 38 years, Nigeria).

Another resident in the UK supported this theme; additionally, she mentioned the need for governments to involve credible organizations that engage different groups in designing messages to improve vaccination uptake. 
"The governments are coming from a difficult position as trust in them is so low. They should hand over the responsibility to credible and honest sources such as Public Health England (PHE) - the PHE should talk to different groups and find what their barriers and beliefs are and involve those groups in designing messages to improve vaccination uptake..." (Female, 49 years, the UK).

\section{Discussion}

We conducted a qualitative study using open-ended questions with a heterogeneous sample of 60 participants who were residents in the UK and Nigeria. Our study focused on understanding the underlying factors in the willingness to receive and barriers to receiving the COVID-19 vaccine from the perspectives of participants. The first question asked participants to describe the experiences they had when they received the vaccine. Participants (only two) who received the vaccine reported adverse bodily reactions (e.g., soreness and itchiness) after receiving the vaccine. Adverse bodily reactions, indeed, are common with many if not all vaccines (Haynes et al., 2020; McClung et al., 2020; Zhu et al., 2020). For this reason, research and development of all pharmaceuticals include all effects of the product, including good and bad.

Our second question asked participants who received the COVID-19 vaccine to tell us what motivated them to receive the vaccine. The participants reported the availability of the vaccine as their core motive for receiving it. This result partly aligned with Danchin et al. (2020) who reported that the availability of the COVID-19 vaccine could optimize its uptake in Australia. However, this finding partly challenged Su et al., (2020) who highlighted that the availability of the COVID-19 vaccine does not translate to its uptake. Therefore, we may argue that apart from the availability of the COVID-19 vaccine, our participants probably received the vaccine because of the perceived benefits that they associated with it as opined by the HBM that guided our current study (Green et al., 2020).

Our third question asked participants who had not received the COVID-19 vaccine to discuss the factors that could determine their willingness to receive the vaccine. Five themes were created from the responses of participants to this question; "concerns about the side/adverse effect", "the perceived benefit of receiving the vaccine", "mistrust (in the pharmaceutical companies that produced the vaccine, the vaccine itself, or governments)", "the need for clarity of information on the vaccine", and "moral obligation to receive the vaccine". The theme of "concerns about the side/adverse effect" revealed that participants were concerned about any possible side/adverse effects that were associated with receiving the vaccine. For instance, a participant endorsing this theme was concerned about the vaccine affecting her chances of getting pregnant in the future. This theme partly supports a related study in the USA with 316 respondents (Pogue et al., 2020). The study found that although $68 \%$ of the participants were supportive of the COVID-19 vaccine, the side/adverse effect of the vaccine remained a concern.

The theme of "the perceived benefit of receiving the vaccine" indicated that many participants' willingness to receive the vaccine was determined by the benefits that they associated with the vaccine. This theme endorsed the studies of Pogue et al. (2020) and Guidry et al. (2021) that were conducted in the USA. Additionally, this theme endorsed a study conducted with 5114 UK adults (Freeman et al., 2020) and a study in New Zealand (Dodd, et al., 2020). These studies reported that the collective importance or benefit associated with receiving the COVID-19 vaccine determined respondents' willingness to receive the vaccine. Theoretically, this theme supported the HBM which states that people engage in behaviors that they perceive will be beneficial to their health (Green et al., 2020).

The theme of "mistrust (in the pharmaceutical companies that produced the vaccine, the vaccine itself, or governments)" was the third theme found in the factors that determined the willingness to receive the vaccine among participants who were not vaccinated. According to this theme, participants had mistrust in the pharmaceutical company that produced the vaccine, the vaccine itself, or their governments. A Nigerian study reported that mistrust in the COVID-19 vaccine may build up in the general public, and this can affect their willingness to get vaccinated (Enitan et al., 2020). Reports from scholars in France and Canada have also highlighted that mistrust can determine vaccine hesitancy (Verger \& Dubé, 2020). Additionally, a global survey with respondents from 19 countries (e.g., France, the USA, the UK, Italy, Nigeria, South Africa, etc.) found that trust in the government was critical for the uptake of the COVID-19 vaccine (Lazarus et al., 2020).

The theme of "the need for clarity of information on the COVID-19 vaccine" was the next theme found in our study. According to this theme, it was reported that participants need clear and accurate information on the vaccine to encourage them to get vaccinated. This agreed with a Nigerian study (Enitan et al., 2020). The study concluded that misinformation and disinformation were important factors that determined the willingness to receive the COVID-19 vaccine among the general public.

The theme of "moral obligation to receive the vaccine" was the last theme found in the factors that determined the willingness to receive the COVID-19 vaccine among participants that had not been vaccinated. This theme aligned with a report from the UK which highlighted that individuals 
have a moral responsibility to get vaccinated (Giubilini, 2020). Another researcher reported that due to moral considerations, healthcare workers considered adopting appropriate behaviors to contain the spread of COVID-19 (Coulthard, 2020).

Our fourth question asked participants who had not received the vaccine to describe the barriers that potentially limited them from receiving the vaccine, apart from the factors that they earlier mentioned as determinants of their willingness. From their responses, three themes were found: "Unavailability of the vaccine in the country of residence", "non-membership to a high-risk group", and "membership to a minority group". The theme of "unavailability of the vaccine in the country of residence" revealed that most participants indicated that the vaccine was unavailable in their countries of residence. This agreed with Reiter et al. (2020) who reported the unavailability of the COVID-19 vaccine as a potential barrier to vaccination. The theme of "nonmembership to a high-risk group" was the second theme. It revealed that participants who were not classified as highrisk groups perceived their non-classification as a potential barrier to their uptake of the vaccine. This supported the study of Williams et al. (2020) which found that membership to high-risk groups may impact the uptake of the COVID-19 vaccine.

The theme of "membership to a minority group" was the last theme reported as a potential barrier according to our participants. This theme described the impact that existing inequalities may have on the COVID-19 vaccine rollout. This theme reinforced the need for ethical consideration with regard to the COVID-19 vaccine rollout (McClung et al., 2020). Additionally, scholars in Portugal have mentioned the need for mitigating the potential impact that inequalities in the healthcare system may have on efforts aimed at containing the spread of COVID-19 (Shaaban et al., 2020).

Our fifth question asked all participants to recommend the efforts that governments can implement to encourage the public uptake of the COVID-19 vaccine. Five themes were created from their responses: "Provide clear information on the COVID-19 vaccine", "endorsement by public figures", "make the vaccine free to receive", "introduce rewards and punishments", and "honesty from governments". The first theme "provide clear information on the COVID-19 vaccine" revealed that participants need their governments to provide clear and accurate information on the COVID-19 vaccine (e.g., governments should educate the general public about the vaccine using layman's terms). This theme endorsed a Nigerian study which found that misinformation and disinformation may play a role in the number of people receiving the vaccine (Enitan et al., 2020). The theme of "endorsement by public figures" was the second recommendation from participants. According to this theme, participants recommended that if public figures are publicized receiving the vaccine, it may thus motivate the general public to receive the vaccine. This theme supported what was documented by Bokemper et al. (2020). The scholars documented that public endorsement can facilitate the uptake of the COVID-19 vaccine.

The next theme was "make the vaccine free to receive". The theme revealed that if the COVID-19 vaccine was free to receive, the number of people receiving the vaccine may increase in number. This theme reinforced the findings of a cross-sectional survey conducted in Malaysia (Wong et al., 2020). The study reported that the affordability of the COVID-19 vaccine and other socioeconomic factors could impact the uptake of the vaccine.

The next theme was "introduce rewards and punishments". According to the participants, if rewards and punishments are attached to the COVID-19 vaccine, it may increase the number of people receiving it. Theoretically, we argued that this theme was a consensus with the operant conditioning of Skinner (1963) which argued that the strength of behavior rests on its consequences (e.g., rewards or punishments). Additionally, this theme supported the study of Freeman et al. (2020). The study reported that the perceived importance (e.g., rewards) of receiving the COVID-19 vaccine may encourage the general public to receive the vaccine.

The last theme found was "honesty from governments". From the perspective of our participants, governments should employ honesty to encourage the general public to receive the vaccine. For instance, a UK resident endorsing this theme highlighted the need for governments to employ credible and honest sources to talk to different groups and find what their barriers are to receiving the COVID-19 vaccine. Again, this theme agreed with the study of Freeman et al. (2020). The study highlighted that honesty and transparency may encourage public uptake of the COVID-19 vaccine.

Our study has some strengths and limitations that must be acknowledged. First, qualitative research is valuable to complement and stand alongside quantitative researches (Wisdom \& Creswell, 2013). Therefore, we hope that our findings will complement the findings from existing quantitative studies (e.g., Enitan et al., 2020; Freeman et al., 2020; Wong et al., 2020). Further, we recruited 60 participants (age range of 18-79 years) residing in two countries, and our thematic analysis conducted after we reached data saturation revealed that specific themes were rarely evident according to participants' country of residence. This, therefore, strengthens the transferability of our results. Nevertheless, we acknowledge that qualitative findings have limited generalizability.

Only two participants (who were UK residents) reported having received the COVID-19 vaccine, and this limited a social reflection of the main driving forces to receiving the vaccine. The last limitation of our study was that our 
participants were recruited from social media using a snowball sampling technique. Therefore, with this form of selection bias, we cannot conclude that their views represent the views of digitally excluded people.

\section{Implication for Practice}

To the best of our knowledge, our study is among the first qualitative studies to explore factors underlying the willingness to receive and barriers to receiving the COVID-19 vaccine. Our findings generated practical implications that were highlighted. First, adverse bodily reactions, indeed, are common with many if not all vaccines. For this reason, clinical trials should be rigorously conducted to find ways of minimizing the possible adverse bodily reactions that may be associated with receiving the COVID-19 vaccine, and results from the clinical trials should be communicated to the general public using "layman's terms". Second, governments through credible healthcare organizations should recruit members of the general public with different demographics, and ask them about their beliefs about the COVID-19 vaccine and their barriers to receiving the COVID-19 vaccine. Further, the recruited members of the general public and their data should be included in the development and implementation of public health education programs that aim to enlighten the general public on the COVID-19 vaccine (both the positive and negative sides of the vaccine) using simple or less complicated words.

Third, there is a need for public figures to publicly endorse the COVID-19 vaccine to reduce mistrust or doubts in the general public. Fourth, governments should be thorough enough to mitigate the inequalities (e.g., membership to minority groups) that present as barriers to receiving the COVID-19 vaccine among the general public. Fifth, COVID-19 vaccination should be affordable to the general public given the differences in socioeconomic status among the general public. Sixth, governments may introduce rewards to strengthen the public uptake of the COVID-19 vaccine. However, governments must be ethically cautious to ensure that any reward is not a breach of the rights of the general public. Last, governments in countries where the COVID-19 vaccine is unavailable should make active efforts to make the vaccine available.

\section{Conclusion}

We concluded that our participants have many factors that determined their willingness to receive the COVID-19 vaccine. We also concluded that apart from the factors that determined their willingness, many other factors present as barriers to receiving the COVID-19 vaccine among them. Additionally, our participants recommended in their own words, various efforts that the governments can implement to strengthen the public uptake of the vaccine. Therefore, considering the themes found in our study and their implications may assist the governments, stakeholders, and healthcare organizations to design appropriate intervention programs that strengthen the public uptake of the COVID-19 vaccine.

Further studies are needed with digitally excluded people because we cannot conclude that the factors underlying their willingness to receive, and barriers to receiving the COVID19 vaccine, were reflected in our data. Finally, future studies should explore the experiences of receiving and motives for receiving the COVID-19 vaccine among people already vaccinated. This is because our study had limited data in this group.

Acknowledgments We would like to thank all participants for giving up their time to participate in our study and provide feedback. We would also like to thank our following colleagues for their contributions: Vivian Dike, Chioma C. Harbor, Lydia E. Adzimah, Amogelang Chepape, Dr. Aminat O. Amusa, Chinyereugo Onyekwere, and James M. Clay.

Author's Contributions Okoloba conceived the study and discussed it with Ogueji who translated it to a researchable idea. Okoloba wrote the introduction, while Ogueji designed the study and wrote the methods. Both authors collected the data, analyzed the data, wrote the discussion, edited the paper, read, and approved the final paper. Therefore, both authors contributed equally and are the first authors of this paper.

Funding We self-funded this study.

Data Availability The data associated with this study are available from the corresponding author upon request.

\section{Declarations}

Conflict of Interest We have no conflict of interest to declare.

Ethical Considerations All procedures performed in our research involving human participants followed the ethical standards of the institutional and/or national research ethics committee, and the Helsinki 1964 ethical declaration, its later amendment, or a comparable standard. An online consent form was utilized to obtain consent from all participants, and all participants consented that findings from their data should be published in this paper.

\section{References}

Beaumont, P. (18 November 2020). "Covid-19 vaccine: who are countries prioritizing for first doses?" The Guardian. ISSN 0261-3077. Retrieved from Covid-19 vaccine: who are countries prioritising for first doses? | World news I The Guardian.

Bokemper, S. E., Huber, G. A., Gerber, A. S., James, E. K., \& Omer, S. B. (2020). Timing of COVID-19 vaccine approval and 
endorsement by public figures. Research Square, https://doi.org/ 10.21203/rs.3.rs-95823/v1.

Booth, A., Hannes, K., Harden, A., Noyes, J., Harris, J., \& Tong, A. (2014). COREQ (consolidated criteria for reporting qualitative studies). In D. Moher, D. G. Altman, K. F. Schulz, I. Simera, \& E. Wager (Eds.), Guidelines for reporting health research: A user's manual (pp. 214-226). John Wiley.

Chen, B., Li, Q. X., Zhang, H., Zhu, J. Y., Wu, Y. H., Xiong, J., et al. (2020). The psychological impact of COVID-19 outbreak on medical staff and the general public. Current Psychology. https:// doi.org/10.1007/s12144-020-01109-0

Coulthard, P. (2020). Dentistry and coronavirus (COVID-19) - moral decision-making. British Dental Journal, 228, 503-505. https:// doi.org/10.1038/s41415-020-1482-1

Cuschieri, S. (2020). COVID-19 panic, solidarity and equity-The Malta exemplary experience. Journal of Public Health. https:// doi.org/10.1007/s10389-020-01308-w

Danchin, M., Biezen, R., Manski-Nankervis, J. A., Kaufman, J., \& Leask, J. (2020). Preparing the public for COVID-19 vaccines: How can general practitioners build vaccine confidence and optimise uptake for themselves and their patients? Australian Journal of General Practice, 49(10), 625.

Dodd, R. H., Pickles, K., Nickel, B., Cvejic, E., Ayre, J., Batcup, C., et al. (2020). Concerns and motivations about COVID-19 vaccination. The Lancet Infectious Diseases. https://doi.org/10.1016/ S1473-3099(20)30926-9

Elliott, R., \& Timulak, L. (2005). Descriptive and interpretive approaches to qualitative research. In J. Miles \& P. Gilbert (Eds.), A handbook of research methods for clinical and health psychology (pp. 147-159). Oxford University Press.

Enitan, S. S., Oyekale, A. O., Akele, R. Y., Olawuyi, K. A., Olabisi, E. O., \& Nwankiti, A. J. (2020). Assessment of knowledge, perception and readiness of Nigerians to participate in the COVID-19 vaccine trial. International Journal of Vaccines and Immunization, 4, 1-13. https://doi.org/10.16966/2470-9948.123

Freeman, D., Loe, B. S., Chadwick, A., Vaccari, C., Waite, F., Rosebrock, L., et al. (2020). COVID-19 vaccine hesitancy in the UK: The Oxford coronavirus explanations, attitudes, and narratives survey (OCEANS) II. Psychological Medicine, 1-34. https://doi. org/10.1017/S0033291720005188

Giubilini, A. (2020). Vaccination ethics. British Medical Bulletin, ldaa036. https://doi.org/10.1093/bmb/ldaa036

Goldman, R. D., Yan, T. D., Seiler, M., Cotanda, C. P., Brown, J. C., Klein, E. J., et al. (2020). Caregiver willingness to vaccinate their children against COVID-19: Cross sectional survey. Vaccine, 38(48), 7668-7673. https://doi.org/10.1016/j.vaccine.2020.09.084

Green, E. C., Murphy, E. M., \& Gryboski, K. (2020). The health belief model. The Wiley Encyclopedia of Health Psychology, 211-214. https://doi.org/10.1002/9781119057840.ch68.

Guidry, J. P., Laestadius, L. I., Vraga, E. K., Miller, C. A., Perrin, P. B., Burton, C. W., et al. (2021). Willingness to get the COVID-19 vaccine with and without emergency use authorization. American Journal of Infection Control, 49(2), 137-142. https://doi.org/10. 1016/j.ajic.2020.11.018

Haynes, B. F., Corey, L., Fernandes, P., Gilbert, P. B., Hotez, P. J., Rao, S., et al. (2020). Prospects for a safe COVID-19 vaccine. Science Translational Medicine, 12(568). https://doi.org/10.1126/ scitranslmed.abe 0948

Kaur, S. P., \& Gupta, V. (2020). COVID-19 Vaccine: A comprehensive status report. Virus Research, 198114. https://doi.org/10.1016/j. virusres.2020.198114

Kreps, S., Prasad, S., Brownstein, J. S., Hswen, Y., Garibaldi, B. T., Zhang, B., \& Kriner, D. L. (2020). Factors associated with US adults' likelihood of accepting COVID-19 vaccination. JAMA Network Open, 3(10), e2025594-e2025594. https://doi.org/10. 1001/jamanetworkopen.2020.25594
Lazarus, J. V., Ratzan, S. C., Palayew, A., Gostin, L. O., Larson, H. J., Rabin, K., et al. (2020). A global survey of potential acceptance of a COVID-19 vaccine. Nature Medicine, 1-4. https://doi.org/10. 1038/s41591-020-1124-9

McClung, N., Chamberland, M., Kinlaw, K., Matthew, D. B., Wallace, M., Bell, B. P., et al. (2020). The advisory committee on immunization practices' ethical principles for allocating initial supplies of COVID-19 vaccine-United States, 2020. Morbidity and Mortality Weekly Report, 69(47), 1782. https://doi.org/10. 15585/2Fmmwr.mm6947e3

Murphy, J., Vallières, F., Bentall, R. P., Shevlin, M., McBride, O., Hartman, T. K., ... \& Hyland, P. (2021). Psychological characteristics associated with COVID-19 vaccine hesitancy and resistance in Ireland and the United Kingdom. Nature Communications, 12(1), 1-15. https://doi.org/10.1038/ s41467-020-20226-9

Neumann-Böhme, S., Varghese, N. E., Sabat, I., Barros, P. P., Brouwer, W., van Exel, J., ... \& Stargardt, T. (2020). Once we have it, will we use it? A European survey on willingness to be vaccinated against COVID-19. European Journal of Health Economics, 21,977-982. https://doi.org/10.1007/s10198-020-01208-6

Nkengasong, J. N., Ndembi, N., Tshangela, A., \& Raji, T. (2020). COVID-19 vaccines: how to ensure Africa has access. Nature, 586, 197-199. https://doi.org/10.1038/ d41586-020-02774-8

Ogueji, I. A., Agberotimi, S. F., Adesanya, B. J., \& Gidado, T. N. (2021a). Mental health and coping strategies during the COVID-19 pandemic: A qualitative study of unemployed and employed people in Nigeria. Analyses of Social Issues and Public Policy. https://doi.org/10.1111/asap.12259

Ogueji, I. A., Okoloba, M. M., \& Demoko Ceccaldi, B. M. (2021b). Coping strategies of individuals in the United Kingdom during the COVID-19 pandemic. Current Psychology. https://doi.org/ 10.1007/s 12144-020-01318-7

Okoloba, M. M., Ogueji, I. A., Darroch, S. J., \& Ogueji, A. M. (2020). A multinational pilot study on the lived experiences and mental health impacts from the COVID-19 pandemic. Global Psychiatry, 3(2), 211-226. https://doi.org/10.2478/ gp-2020-0015

Okoloba, M. M., \& Ogueji, I. A. (2020). Exploring lay people's beliefs about psychology in the United Kingdom (UK). International Journal of Social Sciences \& Educational Studies, 7(2), 40-45. https://doi.org/10.23918/ijsses.v7i2p40

Pogue, K., Jensen, J. L., Stancil, C. K., Ferguson, D. G., Hughes, S. J., Mello, E. J., et al. (2020). Influences on attitudes regarding potential COVID-19 vaccination in the United States. Vaccines, 8(4), 582. https://doi.org/10.3390/vaccines 8040582

Qian, M., \& Jiang, J. (2020). COVID-19 and social distancing. Journal Public of Health. https://doi.org/10.1007/s10389-020-01321-z

Reiter, P. L., Pennell, M. L., \& Katz, M. L. (2020). Acceptability of a COVID-19 vaccine among adults in the United States: How many people would get vaccinated? Vaccine, 38(42), 6500-6507. https:// doi.org/10.1016/j.vaccine.2020.08.043

Ritchie, H., Ortiz-Ospina, E., Beltekian, D., Mathieu, E., Hasell, J., Macdonald, B., ... \& Roser, M. (2021). Coronavirus (COVID-19) vaccinations. Retrieved from Coronavirus (COVID-19) Vaccinations - Statistics and Research - Our World in Data.

Robinson, E., \& Daly, M. (2020). Explaining the rise and fall of psychological distress during the COVID-19 crisis in the United States: Longitudinal evidence from the understanding America study. British Journal of Health Psychology. https://doi.org/10. 1111/bjhp. 12493

Robles-Bello, M. A., Sánchez-Teruel, D., \& Naranjo, N. V. (2020). Variables protecting mental health in the Spanish population affected by the COVID-19 pandemic. Current Psychology, 1-12. https://doi.org/10.1007/s12144-020-01132-1 
Shaaban, A. N., Peleteiro, B., \& Martins, M. R. O. (2020). COVID19: What is next for Portugal? Frontiers in Public Health, 8, 392. https://doi.org/10.3389/fpubh.2020.00392

Sidhu, T., Lemetyinen, H., \& Edge, D. (2020). 'Diabetes doesn't matter as long as we're keeping traditions alive': A qualitative study exploring the knowledge and awareness of type 2 diabetes and related risk factors amongst the young Punjabi Sikh population in the UK. Ethnicity \& Health, 1-19. https://doi.org/10.1080/13557 858.2020 .1827141

Skinner, B. F. (1963). Operant behavior. American Psychologist, 18(8), $503 \mathrm{https} / / /$ psycnet.apa.org/doi/10.1037/h0045185

Su, Z., Wen, J., Abbas, J., McDonnell, D., Cheshmehzangi, A., Li, X., ... \& Cai, Y. (2020). A race for a better understanding of COVID19 vaccine non-adopters. Brain, Behavior, \& Immunity-Health, 100159. https://doi.org/10.1016/j.bbih.2020.100159

Tufford, L., \& Newman, P. (2012). Bracketing in qualitative research. Qualitative Social Work, 11(1), 80-96. $10.1177 \% 2$ F1473325010368316.

Verger, P., \& Dubé, E. (2020). Restoring confidence in vaccines in the COVID-19 era. Expert Review of Vaccines, 19(11), 991-993. https://doi.org/10.1080/14760584.2020.1825945

Warren, N., Kisely, S., \& Siskind, D. (2020). Maximizing the uptake of a COVID-19 vaccine in people with severe mental illness: A public health priority. JAMA Psychiatry. https://doi.org/10.1001/ jamapsychiatry.2020.4396

Williams, L., Gallant, A. J., Rasmussen, S., Brown Nicholls, L. A., Cogan, N., Deakin, K., et al. (2020). Towards intervention development to increase the uptake of COVID- 19 vaccination among those at high risk: Outlining evidence-based and theoretically informed future intervention content. British Journal of Health Psychology, 25(4), 1039-1054. https://doi.org/10.1111/ bjhp. 12468

Wisdom, J., \& Creswell, J. W. (2013). Mixed methods: Integrating quantitative and qualitative data collection and analysis while studying patient-centered medical home models. Rockville: Agency for Healthcare Research and Quality. Available at: http:// pcmh.ahrq.gov/MixedMethods.

Wong, L. P., Alias, H., Wong, P. F., Lee, H. Y., \& AbuBakar, S. (2020). The use of the health belief model to assess predictors of intent to receive the COVID-19 vaccine and willingness to pay. Human Vaccines \& Immunotherapeutics, 16(9), 2204-2214. https://doi. org/10.1080/21645515.2020.1790279

World Health Organization. (2020). Coronavirus disease 2019 (COVID-19): situation report, 82.

Zhu, F. C., Guan, X. H., Li, Y. H., Huang, J. Y., Jiang, T., Hou, L. H., et al. (2020). Immunogenicity and safety of a recombinant adenovirus type-5-vectored COVID-19 vaccine in healthy adults aged 18 years or older: A randomised, double-blind, placebo- controlled, phase 2 trial. The Lancet, 396(10249), 479-488. https:// doi.org/10.1016/S0140-6736(20)31605-6

Publisher's Note Springer Nature remains neutral with regard to jurisdictional claims in published maps and institutional affiliations. 